\title{
Inequity in healthcare needs, health service use and financial burden of medical expenditures in China: results from a consecutive household monitoring study in Jiangsu Province
}

Weixi Jiang ${ }^{1}$, Xiaolin $X_{u^{2}}$, Shenglan Tang ${ }^{3}$, Ling X ${ }^{4}$, Yaoguang Zhang ${ }^{5}$, Chris Elbers ${ }^{6}$, Frank Cobelens ${ }^{7}$ and Lijing $\operatorname{Yan}^{8^{*}}$ (D)

\begin{abstract}
Background: Although public medical insurance covers over 95\% of the population in China, disparities in health service use and out-of-pocket (OOP) health expenditure across income groups are still widely observed. This study aims to investigate the socio-economic disparities in perceived healthcare needs, informal care, formal care and payment for healthcare and explore their equity implication.

Methods: We assessed healthcare needs, service use and payment in 400 households in rural and urban areas in Jiangsu, China, and included only the adult sample $(N=925)$. One baseline survey and 10 follow-up surveys were conducted during the 7-month monitoring period, and the Affordability Ladder Program (ALP) framework was adopted for data analysis. Negative binomial/zero-inflated negative binomial and logit regression models were used to explore factors associated with perceived needs of care and with the use of self-treatment, outpatient and inpatient care respectively. Two-part model and logit regression modeling were conducted to explore factors associated with OOP health expenditure and with the likelihood of incurring catastrophic health expenditure (CHE).

Results: After adjusting for covariates, rural residence was significantly associated with more perceived healthcare needs, more self-treatment, higher probability of using outpatient and inpatient service, more OOP health expenditure and higher likelihood of incurring catastrophic expenditure $(P<0.05)$. Compared to the Urban Employee Basic Medical Insurance (UEBMI), enrollment in the New Rural Cooperative Medical Scheme (NRCMS) or in the Urban Resident Basic Medical Insurance (URBMI) was correlated with lower probability of ever using outpatient services, but with more outpatient visits when people were at risk of using outpatient service $(P<0.05)$. NRCMS/URBMI enrollment was also associated with higher likelihood of incurring CHE compared to UEBMI enrollment $(\mathrm{OR}=2.02, P<0.05)$; in stratified analysis of the rural and urban sample this effect was only significant for the rural population.

Conclusions: The rural population in Jiangsu perceived more healthcare needs, had a higher probability of using both informal and formal healthcare services, and had more OOP health expenditure and a higher likelihood of incurring CHE. The inequity mainly exists in health care financing, and may be partially addressed through improving the benefit packages of NRCMS/URBMI.
\end{abstract}

Keywords: Equity in healthcare, Healthcare needs, Health service use, Healthcare financing

\footnotetext{
* Correspondence: lijing.yan@dukekunshan.edu.cn

${ }^{8}$ Global Health Research Center, Duke Kunshan University, No. 8 Duke

Avenue, Kunshan 215316, China

Full list of author information is available at the end of the article
}

(c) The Author(s). 2019 Open Access This article is distributed under the terms of the Creative Commons Attribution 4.0 International License (http://creativecommons.org/licenses/by/4.0/), which permits unrestricted use, distribution, and reproduction in any medium, provided you give appropriate credit to the original author(s) and the source, provide a link to the Creative Commons license, and indicate if changes were made. The Creative Commons Public Domain Dedication waiver (http://creativecommons.org/publicdomain/zero/1.0/) applies to the data made available in this article, unless otherwise stated. 


\section{Background}

The Chinese health care system has experienced rapid changes along with socio-economic reform. On the supply side, the government has in recent years been investing in health care infrastructure, especially in primary health care institutions [1]. On the demand side, three public health insurance schemes, the Urban Employee Basic Medical Insurance (UEBMI), the Urban Resident Basic Medical Insurance (URBMI) and the New Rural Cooperative Medical Scheme (NRCMS), were gradually established since late 1990s. Currently over 95\% of the Chinese population are covered by these three public insurance schemes, leaving less than 5\% not covered by any insurance scheme, and medical services are mostly financed through the co-payment mechanism of the insurance schemes [2]. Inpatient medical services are covered with reimbursement rates ranging from 50 to $90 \%$ for different schemes at different levels of hospitals, but the reimbursement rates for outpatient services are still rather low for URBMI and NRCMS [3, 4].

Many studies have assessed equity in health care utilization and financing in terms of insurance types and income. Recent studies on the three public insurance schemes generally show that they improved people's access to formal care, including both outpatient and inpatient services, and narrowed the gaps in service use across income groups with the expansion of insurance coverage [3, 5-8]. Nevertheless, one report from the World Bank showed that the separation in the management of the financing and benefit packages of health insurance schemes across insurance types and regions weakened the risk-pooling effect, and caused inequity problems [9]. The high proportion of out-of-pocket (OOP) health expenditure also indicated obstacles to accessing health care and high financial burden of health care [10-12]. For each type of insurance specifically, research on URBMI and UEBMI reveals that these schemes benefited patients from higher income groups more, as poorer patients were less likely to use expensive care, thus receiving less reimbursement than the welloff, and the urban insurance systems failed to reduce the OOP health expenditure for the disadvantaged group [6, $13,14]$. For NRCMS studies show that the expansion of NRCMS narrowed the gaps in inpatient service use and encouraged poorer patients to seek informal and preventive care. However they also showed that the care utilization pattern under NRCMS was still pro-rich and that NRCMS did not lower health expenditure nor provide sufficient protection for the poor $[7,8,15]$.

A rich literature also explored inequity in health care regarding a series of demographic and socio-economic factors. Several recent studies revealed that with the expansion of insurance coverage, the rural-urban disparities in health service utilization and reimbursement rate substantially narrowed in recent years, but still exist [16-18]. Rural patients enjoyed lower reimbursements and bore a high burden of medical expenditure with reference to their income level [17, 19]. Studies also showed how ethnic minority, income, education and insurance coverage may have a differential impact on the service use pattern of rural and urban populations [20, 21]. Nevertheless, there is a lack of systematic analysis on how demographic and socio-economic factors would impact on the whole health seeking process under the current health care system, starting from health care needs to payment for services for both rural and urban population.

In this study we referred to the Affordability Ladder Program (ALP) which provides a holistic approach to examining equity in the health care system from the demand-side perspective through step-by-step analysis, taking into account the perceived health care needs, informal care, formal care and payment for health care [22]. Several studies have used this framework to explore access to and payment for healthcare services in other countries [22-25]. This framework also allowed the exploration of the potential differential or synergistic impact of a certain factor on health equity in each step of the entire health care seeking path, which was not possible in most previous studies and were the main original contribution of our research. This study was located in Jiangsu, a relatively well-developed province in eastern China with a per capita GDP of $\$ 14,000$ in 2015 , where over $95 \%$ of the population were covered by one of the three public health insurance schemes [26]. We focused on the equity implication of non-need factors for health care such as residence, income and insurance coverage [27], and explored how these factors may influence each ladder step of health care for the rural and urban population, respectively.

\section{Methods \\ Sampling design}

One urban district (Gusu) and one rural county (Jinhu) in Jiangsu were selected as study sites. Gusu and Jinhu are located in southern and northern Jiangsu respectively, and the GDP per capita (RMB 136,556) in Gusu is twice as in Jinhu (RMB 65,535). Disproportionate stratified sampling was applied with a sample size of 200 households in each site. A list of households with noncommunicable disease (NCD) patients was obtained from the local health bureau, and 100 households were randomly selected from the list in each site. The other 100 sample households were randomly selected from the rest of the households in the study sites. As the sample size is relatively small, the households with NCD patients were over-sampled in order to increase the total events of service use. The project also aims to look at 
NCD management for which the results are yet to be published.

\section{Data collection}

This study consisted of a baseline survey and 10 followup surveys over 7 consecutive months during 20152016, with the first 6 surveys conducted bi-weekly and the last 4-monthly (the first 6 surveys were in phase 1, the remaining 4 were in phase 2 when survey frequency was reduced with regard to respondents' feedback that the surveys in phase 1 were too frequent; see Additional file 1 for the Questionnaire). One knowledgeable person from each household, usually the household head, signed informed consent before the interview, and answered on behalf of all members in the household. The baseline survey gathered participants' basic demographic, socioeconomic and health information, as well as inpatient service use and expenditure in the past year. Each follow-up survey contained 6 questionnaires concerning chronic disease management for different NCDs, emergent illness (including emergent conditions of NCDs), patients' utilization of self-treatment, outpatient and inpatient services as well as medical costs and out-ofpocket payments.

The baseline survey and the last follow-up survey of phase 1 (6th) and phase 2 (10th) were conducted through face-to-face interviews by trained interviewers in the participants' homes. For other follow-up surveys participants were asked to choose between: 1) filling survey questionnaires themselves; 2) call interviewers when disease/health service use occurred; 3) face-to-face interview at home; or 4) telephone interview for the followup survey. More than $90 \%$ of households chose face-toface interview, a few opted for telephone interview and almost no households filled the survey questionnaires themselves or called interviewers.

All questionnaires were checked by supervisors of the interviewers before transferring to the investigators. For quality assurance, a 5\% sample of the questionnaires were randomly selected and double-checked by investigators through telephone re-interview; the rate of concordance exceeded 95\%. Data was double entered, and inconsistencies, outliers and missing values were also double-checked in order to ensure data quality. Datasets were encrypted in storage and de-identified during data analysis to protect participants' confidentiality.

\section{Data analysis}

We included only the adult population in the analysis as children usually do not make care seeking decisions themselves [28]. Descriptive analyses were conducted to examine the demographic and socio-economic characteristics of the overall sample as well as of the urban and rural sample separately. The chi-squared test and t-test were applied to test for significance of differences between the rural and the urban sample. Based on the ALP framework [22] we identified 6 key outcome variables of interests throughout the care-seeking path as shown in Table 1 . The total of reporting emergent illness episodes, conducting self-treatment, outpatient service use, inpatient service use, and the total amount of OOP health expenditure were aggregated over the 7-month survey period. Catastrophic health expenditure was defined as total OOP health expenditure exceeding $10 \%$ of household income [29]. The data were analyzed using STATA 13.1(StataCorp, Texas, USA).

Multivariate regression models were used to explore factors associated with each of these outcome variables of interests. As preliminary analyses showed that there were many "zeros" in the data (no emergent illness, no service use and no health expenditure), we considered standard Poisson/negative binomial (NB) models versus zero-inflated Poisson (ZIP) /negative binomial (ZINB) models for the first three outcomes which were count variables, and a generalized linear model (GLM) versus a two part model combining logit regression and GLM for the OOP health expenditure [30]. The zero-inflated models have two processes that separately model the likelihood of not being at risk of having the event (process 1, note that a positive coefficient or relative risk $>1$ implies a lower probability of being at risk) and the total number of events given that one is at risk (process 2). The two-part model estimates the likelihood of incurring any OOP medical expenditure and the amount of expenditure if incurred in two steps [30]. As for model selection, we considered the Akaike Information Criterion (AIC) and the Bayesian Information Criterion (BIC) of each model first, and Vuong's closeness test for ZINB vs. the standard negative binomial model if AIC and BIC preferred different models [31]. Based on these criteria we selected the ZINB model for the total episodes of self-reported emergent illness, the NB model for selftreatment, the ZINB model for outpatient service use and two-part models combining logit regression and GLM for OOP health expenditure (see Additional file 2: Table S1). As only 9 patients in our sample were admitted more than once, we coded inpatient service use as a binary variable of use/non-use. Logit regression was conducted to analyze factors associated with the likelihood of ever using inpatient services and incurring $\mathrm{CHE}$.

As for the independent variables, we focused on the effects of factors reflecting socio-economic status (SES), including rural/urban residence, education level, income, employment and the status of health insurance, and adjusted for factors that may affect both these SES factors and the outcomes, including age, gender, marital status and presence of NCDs. Age, income and education level were treated as categorical variables in the regression 
Table 1 Key outcome variables identified based on the ALP framework

\begin{tabular}{ll}
\hline Healthcare seeking path & Outcome variables \\
\hline Perceived health care needs & 1) number of self-reported emergent illness episodes \\
Health service use & 2) self-treatment \\
Informal care & 3) outpatient service use \\
Formal care & 4) inpatient service use \\
Payment for health care & 5) total OOP health expenditure \\
Financial burden of health care & 6) catastrophic health expenditure (CHE) during the survey period
\end{tabular}

models. As for insurance, we grouped people with the new cooperative medical scheme (NRCMS) together with those enrolled in urban resident basic medical insurance (URBMI), as they provided similar benefit packages and only $6 \%$ of the sample were enrolled in URBMI. The average income per capita was divided into 3 groups: the richest $33.3 \%$, middle $33.3 \%$ and poorest $33.3 \%$ for the whole sample. Standard errors were adjusted for household clustering considering the intrahousehold correlation.

\section{Results}

\section{Sample characteristics}

Four-hundred households participated and completed the surveys, totaling 1057 people. We included the 925 adult participants in the analyses. Table 1 shows the demographic, socio-economic, health status and the descriptive analysis of the six outcome variables for the overall sample as well as the samples Gusu (urban) and Jinhu (rural) separately. The sample included 463 adult participants in the urban area and 462 in the rural area. The gender distribution was almost balanced, and $44.1 \%$ were over 60 years old. Participants in the urban area had higher socio-economic status in terms of education, employment and income. In the rural sample $27.9 \%$ of participants had never completed primary school education, while this was only $2.6 \%$ in the urban sample. The average income per capita of the households in the urban sample was almost twice that in the rural sample. Over $95 \%$ of the sample were covered by public health insurance, therefore we could not explore the effects of having no public health insurance on health care utilization. Over $70 \%$ of urban sample were covered by UEBMI while in the rural sample $87.5 \%$ were enrolled in the NRCMS. As for NCD status, $45.7 \%$ of the sampled population had at least one type of NCD, and this rate was slightly higher in the rural area. Descriptive analysis on the six outcome variables showed that the rural sample had more self-reported emergent illness episodes, used both more informal and formal health care services, had higher OOP health expenditure and higher likelihood of incurring $\mathrm{CHE}$.

\section{Factors associated with health care needs}

Table 3 shows the association between perceived health care needs and a series of demographic, health status and socio-economic factors, using the ZINB model. Process 1 of the model showed that, after adjusting for other covariates, people with rural residence were much more likely to be at risk of reporting emergent illness as compared to their urban counterparts $(\mathrm{OR}=0.02,95 \%$ CI: $0.00,0.26)$. Having any NCD also increased the probability of such risk $(\mathrm{OR}=0.33,95 \% \mathrm{CI}: 0.11,0.91)$.

Table 3 Regression analysis of factors associated with selfreported emergent illness episodes using ZINB model

\begin{tabular}{|c|c|c|c|c|}
\hline & \multicolumn{2}{|c|}{ Process 1} & \multicolumn{2}{|c|}{ Process 2} \\
\hline & $\mathrm{OR}$ & $95 \% \mathrm{Cl}$ & IRR & $95 \% \mathrm{Cl}$ \\
\hline \multicolumn{5}{|l|}{ Age } \\
\hline$<30$ & ref. & & ref. & \\
\hline $30-59$ & 0.24 & $0.03,1.81$ & 0.76 & $0.30,1.88$ \\
\hline$>=60$ & 0.41 & $0.05,2.84$ & 0.73 & $0.30,1.78$ \\
\hline Male & 0.88 & $0.31,2.50$ & 0.91 & $0.68,1.24$ \\
\hline Rural residence & 0.02 & $0.00,0.26$ & 0.73 & $0.41,1.29$ \\
\hline Married & 0.76 & $0.28,2.02$ & 0.93 & $0.65,1.32$ \\
\hline \multicolumn{5}{|l|}{ Education Level } \\
\hline no education & ref. & & ref. & \\
\hline primary and junior high & 1.36 & $0.18,9.78$ & 0.86 & $0.64,1.16$ \\
\hline senior high school and above & 0.76 & $0.07,7.22$ & 0.6 & $0.36,1.01$ \\
\hline Employed & 1.17 & $0.36,3.77$ & 1.07 & $0.79,1.43$ \\
\hline \multicolumn{5}{|l|}{ Insurance } \\
\hline UEBMI & ref. & & ref. & \\
\hline NRCMS/URBMI & 1.75 & $0.66,4.65$ & 1.67 & $1.03,2.71$ \\
\hline \multicolumn{5}{|l|}{ Income level } \\
\hline poorest $33.3 \%$ & ref. & & ref. & \\
\hline middle $33.3 \%$ & 0.92 & $0.19,4.29$ & 0.94 & $0.67,1.32$ \\
\hline richest 33.3\% & 0.75 & $0.19,2.86$ & 0.85 & $0.58,1.23$ \\
\hline With NCD & 0.33 & $0.11,0.91$ & 1.39 & $1.02,1.89$ \\
\hline
\end{tabular}

(OR odds ratio, IRR incident rate ratio. Process 1 modeled the likelihood of not being at risk of reporting self-reported illness, process 2 modeled the total number of self-reported emergent illness episodes given that one is at risk. The sample size is the same as described in Table 2. All estimates were adjusted.) 
Process 2 of the model shows that, after adjusting for other factors, having a NCD was in addition associated with reporting more emergent illness episodes (IRR = 1.39, 95\%CI: 1.02, 1.89). People enrolled in NRCMS/ URBMI also tended to report more emergent illness episodes compared to those enrolled in UEBM, (IRR $=1.67$, 95\% CI: 1.03, 2.71). Education level seemed negatively associated with the number of self-reported emergent illness episodes, and the association was almost significant for those with highest education level (senior high school and above).

Factors associated with use of self-treatment, outpatient and inpatient service

Table 4 shows the analyses of the numbers of selftreatments, outpatient service use and inpatient service use for a series of demographic, health status and socioeconomic factors, using different regression models. Multivariable NB regression of self-treatment on these factors showed that older age, rural residence and having NCD were significantly associated with increased use of self-treatment, and the effect was particularly strong for rural residence (IRR $=6.07,95 \% \mathrm{CI}: 2.86,12.88$ ). As for outpatient service use, multivariable regression analysis using the ZINB model showed that rural residence was associated with much higher probability of being at risk of using outpatient services (i.e. using any of these services) compared to urban residence $(\mathrm{OR}=0.02,95 \% \mathrm{CI}$ : $0.00,0.10)$. Conversely, after adjusting for other covariates, enrollment in NRCMS/RBMI significantly decreased the probability of using any outpatient service compared to UEBMI (OR $=13.29,95 \%$ CI: $1.34,132.24)$, which means NRCMS/RBMI may discourage outpatient service use. Nevertheless, for those who were at risk of using outpatient service, NRCMS/RBMI was significantly associated with more use (IRR $=2.75,95 \%$ CI: 1.13 , 6.72). Multivariable logit regression of inpatient service use showed that rural residence and having NCD were associated with higher probability of using inpatient service $(P<0.05)$, while men were less likely to use inpatient service than female $(P<0.05)$.

\section{Out-of-pocket (OOP) payment and financial burden across} income groups

Table 5 shows the results of regression analyses of factors associated with OOP health expenditure using a two-part model combining logit regression and GLM, as well as factors associated with CHE using a logit model. Similar to the results of the analysis on inpatient service use, NCD and rural residence were significantly associated with higher probability of incurring medical expenditure and CHE $(P<0.001)$ after adjusting for other covariates. For those who had out-of-pocket health expenditure, men tended to spend less than women, and
Table 2 Basic characteristics of study participants (\%)

\begin{tabular}{|c|c|c|c|}
\hline & Total sample & Gusu & Jinhu \\
\hline & & (urban) & (rural) \\
\hline & $n=925$ & $n=463$ & $n=462$ \\
\hline \multicolumn{4}{|l|}{ Gender } \\
\hline male & 51.2 & 47.08 & 49.6 \\
\hline \multicolumn{4}{|l|}{ Age } \\
\hline average (sd) & $54.5(17.0)$ & $53.5(18.8)$ & $55.5(15.0)$ \\
\hline $18-29$ & 10.7 & 12.3 & 9.1 \\
\hline $30-59$ & 45.2 & 41.3 & 49.1 \\
\hline$>=60$ & 44.1 & 46.4 & 41.8 \\
\hline \multicolumn{4}{|l|}{ Marriage } \\
\hline married & 85.4 & 85.3 & 85.5 \\
\hline \multicolumn{4}{|l|}{ Education } \\
\hline Below primary & 15.2 & 2.6 & 27.9 \\
\hline primary and junior high & 48.7 & 41 & 56.3 \\
\hline senior high school and above & 36.1 & 56.4 & 15.8 \\
\hline \multicolumn{4}{|l|}{ Employment } \\
\hline employed & 55.0 & 37.8 & 72.3 \\
\hline retired & 27.1 & 50.8 & 3.3 \\
\hline unemployed & 21.4 & 17 & 26.2 \\
\hline \multicolumn{4}{|l|}{ Health insurance } \\
\hline UEBMI & 40.5 & 73.4 & 7.6 \\
\hline URBMI & 6.2 & 7.6 & 4.8 \\
\hline NRCMS & 49 & 10.6 & 87.5 \\
\hline other and no insurance & 4.3 & 8.4 & 0.2 \\
\hline \multicolumn{4}{|c|}{ Mean household income per capita (RMB) } \\
\hline average (sd) & $2135(1395)$ & $2807(1462)$ & $1462(920)$ \\
\hline \multicolumn{4}{|l|}{ With NCD } \\
\hline yes & 45.7 & 43.8 & 47.6 \\
\hline \multicolumn{4}{|c|}{ Self-reported emergent illness episodes } \\
\hline mean (sd) & $0.98(1.66)$ & $0.40(1.12)$ & $1.56(1.90)$ \\
\hline \multicolumn{4}{|l|}{ Total times of self-treatment } \\
\hline mean (sd) & $0.39(0.94)$ & $0.06(0.37)$ & $0.71(1.19)$ \\
\hline \multicolumn{4}{|l|}{ Total times of outpatient service } \\
\hline mean (sd) & $0.66(1.34)$ & $0.35(0.96)$ & $0.97(1.57)$ \\
\hline$\%$ inpatient service use & 6.0 & 3.9 & 8.0 \\
\hline \multicolumn{4}{|l|}{ Total OOP health expenditure } \\
\hline mean (sd) & $594(4266)$ & $201(1940)$ & $991(5697)$ \\
\hline$\% \mathrm{CHE}$ & 16.8 & 6.5 & 27.1 \\
\hline
\end{tabular}

men were also less likely to incur catastrophic expenditure $(P<0.05)$. People in NRCMS/RBMI were also twice likely to incur CHE as those enrolled in UEBMI $(\mathrm{OR}=$ 2.02, 95\% CI: 1.10, 3.73), after adjusting for other variables.

\section{Stratified analysis on the rural and urban sample}

We further explored the effects of demographic and SES factors on these outcomes of interests for urban and 
Table 4 Regression analysis of factors associated with self-treatment, outpatient service and inpatient service use

\begin{tabular}{|c|c|c|c|c|c|c|c|c|}
\hline & \multirow{2}{*}{\multicolumn{2}{|c|}{$\frac{\text { Self-treatment }}{\mathrm{NB}}$}} & \multicolumn{4}{|c|}{ Outpatient service use } & \multirow{2}{*}{\multicolumn{2}{|c|}{$\begin{array}{l}\text { Inpatient service use } \\
\text { logit }\end{array}$}} \\
\hline & & & \multicolumn{2}{|c|}{ ZINB-proc1 } & \multicolumn{2}{|c|}{ ZINB-proc2 } & & \\
\hline & IRR & $95 \% \mathrm{Cl}$ & OR & $95 \% \mathrm{Cl}$ & $\mathrm{IRR}$ & $95 \% \mathrm{Cl}$ & $\mathrm{OR}$ & $95 \% \mathrm{Cl}$ \\
\hline \multicolumn{9}{|l|}{ Age } \\
\hline$<30$ & ref. & & ref. & & & & ref. & \\
\hline $30-59$ & 2.41 & $1.24,4.69$ & 0.64 & $0.13,3.07$ & 0.75 & $0.32,1.75$ & 0.34 & $0.10,1.15$ \\
\hline$>=60$ & 2.27 & $1.10,4.68$ & 0.76 & $0.10,5.93$ & 0.59 & $0.25,1.40$ & 1.11 & $0.31,3.97$ \\
\hline Male & 0.94 & $0.67,1.30$ & 1.66 & $0.48,5.76$ & 0.98 & $0.71,1.37$ & 0.37 & $0.18,0.77$ \\
\hline Rural residence & 6.07 & $2.86,12.88$ & 0.02 & $0.00,0.10$ & 0.56 & $0.17,1.85$ & 3.56 & $1.60,7.93$ \\
\hline Married & 0.87 & $0.57,1.31$ & 0.59 & $0.08,4.13$ & 0.86 & $0.55,1.35$ & 1.85 & $0.75,4.52$ \\
\hline \multicolumn{9}{|l|}{ Education Level } \\
\hline no education & ref. & & ref. & & & & ref. & \\
\hline primary and junior high school & 1.09 & $0.77,1.53$ & 0.5 & $0.05,4.97$ & 0.71 & $0.47,1.08$ & 1.27 & $0.54,2.99$ \\
\hline senior high school above & 0.87 & $0.49,1.54$ & 1.3 & $0.04,39.13$ & 0.71 & $0.33,1.52$ & 1.7 & $0.53,5.57$ \\
\hline Employed & 0.91 & $0.62,1.33$ & 1.45 & $0.28,7.57$ & 1.07 & $0.72,1.58$ & 0.61 & $0.28,1.30$ \\
\hline \multicolumn{9}{|l|}{ Insurance } \\
\hline UEBMI & ref. & & ref. & & & & ref. & \\
\hline NRCMS/URBMI & 1.48 & $0.75,2.91$ & 13.29 & $1.34,132.24$ & 2.75 & $1.13,6.72$ & 1.1 & $0.46,2.62$ \\
\hline \multicolumn{9}{|l|}{ Income level } \\
\hline poorest 33.3\% & ref. & & ref. & & & & ref. & \\
\hline middle $33.3 \%$ & 0.89 & $0.61,1.30$ & 0.26 & $0.02,2.86$ & 0.79 & $0.56,1.12$ & 1.52 & $0.69,3.33$ \\
\hline richest 33.3\% & 0.61 & $0.34,1.09$ & 0.21 & $0.00,13.65$ & 0.71 & $0.42,1.20$ & 1.07 & $0.46,2.50$ \\
\hline With NCD & 1.49 & $1.12,1.99$ & 0.06 & $0.00,4.97$ & 1.21 & $0.80,1.85$ & 2.65 & $1.41,4.95$ \\
\hline
\end{tabular}

rural population separately. Gender played a role in the rural but not in the urban area. Compared to women, men in the rural area tended to report fewer emergent illnesses, use less inpatient and outpatient services, and thus less often incurred catastrophic expenditure. It is also noticeable that for the rural sample, people enrolled in NRCMS/URBMI were more likely to incur CHE compared to those enrolled in UEBMI, and being in the richest tertile also decreased the likelihood of incurring $\mathrm{CHE}$. Nevertheless, insurance category and income were not significantly associated with the possibility of incurring CHE in the urban sample, and only NCD status seemed to have an effect on CHE $(P<0.05)$ (see Additional file 2: Tables S2-S6).

\section{Discussion}

Findings from this study revealed a clear rural-urban distinction: the rural population tended to have more perceived health care needs, had a higher probability of using both informal (self-treatment) and formal (outpatient and inpatient) healthcare services, and had more OOP health expenditure and a higher likelihood of incurring catastrophic expenditure after controlling for other factors. The rural-urban difference in perceived health care needs may be due to unobserved disparities in health status, for example, healthier people are more likely to move to urban areas to seek job opportunities. In our study situated in a developed region, the rural population have access to care upon need, non-need factors such as income seemed to have no effect on health care utilization, and insurance type only had an impact on outpatient service use. Nevertheless, while the expansion of insurance coverage, mainly NRCMS/URBMI, and the investment in health care infrastructure have been narrowing the gaps in service use, people in rural areas are still facing a higher financial burden of treatment.

Besides the rural-urban difference, people enrolled in UEBMI were less likely to incur catastrophic expenditures, and our separate analysis of the rural and urban sample showed that this protective effect is significant for the rural population, but no for the urban population. While previous studies have revealed that current benefit packages of NRCMS are not sufficient to protect people from catastrophic spending [4,32], our study also suggests that we may need to improve the coverage range and reimbursement rate of NRCMS/URBMI to reduce the possibility of catastrophic expenditure, which is particularly urgent when more poor people start to seek 
Table 5 regression analysis of factors associated out-of-pocket health expenditure and CHE

\begin{tabular}{|c|c|c|c|c|c|c|}
\hline & \multicolumn{4}{|c|}{ OOP health expenditure } & \multicolumn{2}{|c|}{ Catastrophic health expenditure } \\
\hline & \multicolumn{2}{|c|}{ part1-logit } & \multicolumn{2}{|c|}{ part2-GLM } & \multicolumn{2}{|l|}{ logit } \\
\hline & OR & $95 \% \mathrm{Cl}$ & Coef. & $95 \% \mathrm{Cl}$ & OR. & $95 \% \mathrm{Cl}$ \\
\hline \multicolumn{7}{|l|}{ Age } \\
\hline$<30$ & ref. & & & & ref. & \\
\hline $30-59$ & 1.46 & $0.73,2.92$ & 57.7 & $-1810.2,1925.7$ & 1.01 & $0.40,2.59$ \\
\hline$>=60$ & 1.07 & $0.51,2.21$ & 1898.1 & $-722.9,4519.0$ & 1.13 & $0.44,2.94$ \\
\hline Male & 0.8 & $0.58,1.11$ & -2207.4 & $-4337.5,-77.3$ & 0.58 & $0.38,0.89$ \\
\hline Rural residence & 6.6 & $3.95,11.03$ & 1094.2 & $-802.5,2991.0$ & 2.92 & $1.61,5.30$ \\
\hline Married & 1.16 & $0.72,1.86$ & 595 & $-620.0,1810.0$ & 0.94 & $0.53,1.67$ \\
\hline \multicolumn{7}{|l|}{ Education Level } \\
\hline no education & ref. & & & & ref. & \\
\hline primary and junior high & 0.68 & $0.42,1.11$ & 1788.4 & $-634.9,4211.7$ & 0.97 & $0.57,1.64$ \\
\hline senior high school and above & 0.62 & $0.32,1.18$ & 2199.7 & $-761.7,5161.1$ & 0.73 & $0.34,1.54$ \\
\hline Employed & 0.9 & $0.58,1.39$ & 712.9 & $-1453.9,2879.8$ & 0.61 & $0.37,1.02$ \\
\hline \multicolumn{7}{|l|}{ Insurance } \\
\hline UEBMI & ref. & & & & ref. & \\
\hline NRCMS/URBMI & 1.33 & $0.8,2.19$ & 238.5 & $-640.6,1117.7$ & 2.02 & $1.10,3.73$ \\
\hline \multicolumn{7}{|l|}{ Income level } \\
\hline poorest 33.3\% & ref. & & & & ref. & \\
\hline middle $33.3 \%$ & 1.06 & $0.69,1.61$ & -237.4 & $-1661.7,1186.8$ & 0.72 & $0.43,1.20$ \\
\hline richest 33.3\% & 1.09 & $0.69,1.74$ & 877.2 & $-2167.2,3921.6$ & 0.57 & $0.31,1.05$ \\
\hline With NCD & 1.99 & $1.42,2.78$ & 212.8 & $-1245.0,1670.6$ & 2.97 & $1.93,4.58$ \\
\hline
\end{tabular}

(Part 1 of the two-part model used logit regression to estimate the likelihood of incurring OOP health expenditure, and part 2 used GLM to model the amount of OOP health expenditure if occurred. All estimates were adjusted.)

care. We also noticed that while enrollment in NRCMS/ RBMI indicates lower probability of using any outpatient care, it was associated with increased numbers of visits for those who were at risk of using outpatient service. This finding suggests that as NRCMS/URBMI provides little coverage for outpatient services, people may delay care seeking until the disease is serious, which may in turn lead to higher expenditure for treatment.

As the health care reform in China continues, NRCMS is being, or has been, integrated with URBMI in many regions. Several studies on this integration show that it narrowed the rural-urban gaps in inpatient benefit, improved the quality of health care and reduced the health care expenditure of the rural population [33, 34]. The integration of all the three public insurances has just started to be piloted in some cities [35]. Although some studies have revealed that such integration would encounter administrative and technical challenges [36, 37], it is still regarded by many researchers as a critical way of reducing inequity across insurance schemes and regions $[9,37,38]$. In our study we emphasize that there is a need to reduce the gaps in benefit packages for UEBMI and NRCMS/URBMI, in view of the increasing health care demands from the rural population.
In this study we investigated and identified the inequity in health care needs, service use and financing between rural and urban population, as well as across different types of public insurance. Nevertheless, this study also has several limitations. As mentioned above, it was conducted in the most developed eastern area of China where in 2017 less than $0.8 \%$ of the rural population still lived in absolute poverty [39]. Findings from this study may underestimate the level of inequity in health care with regards to the overall situation in China, as poorer people in this area were still able to access inpatient care despite of the high OOP medical expenditure. External validation of the results was also not possible as we only have data in Jiangsu. The short monitoring period and changes in the frequency of follow-up surveys also restricted us in observing seasonal changes in health care needs or service use. Besides, CHE is a household-level variable and we realize that when we use individual regressors as proxy for their householdlevel equivalents, we introduced measurement error 'on the right-hand side', leading to attenuation bias. On the other hand, the fact that we included all adults from a household in our ample tends to neutralize this bias. In light of these findings and limitations, future research 
may increase the number of study sites and extend the length of monitoring to get a more complete understanding of the equity issues in health care across regions in China at different developmental stages. Besides, the reason why men in rural area made less use of inpatient services remains unclear, and deserves further investigation.

\section{Conclusion}

The rural population in Jiangsu perceived more health care needs and had a higher probability of using both informal and formal health care services than the urban population. Rural population also had higher OOP health expenditure, and NRCMS /URBMI provided less sufficient protection from catastrophic expenditure as compared to UEBMI. While the expansion of the coverage in NRCMS/URBMI has narrowed the gaps in health care utilization, the inequities in health care financing may be further addressed through improving the benefit packages of NRCMS/URBMI.

\section{Supplementary information}

Supplementary information accompanies this paper at https://doi.org/10. 1186/s12913-019-4796-4.

Additional file 1: Questionnaire Survey questionnaire for this research project.

Additional file 2. Results of the model comparison using the Akaike Information Criterion (AIC), the Bayesian Information Criterion (BIC) and the Vuong's closeness test, and the stratified analysis on the rural and urban sample. Table S1. The AIC, BIC and results of Vuong test of different regression models for each outcome variable Table S2. Regression analysis of factors associated with self-reported emergent illness using NB model: rural vs urban. Table S3. Regression analysis of factors associated with the total times of conducting self-treatment using NB model: rural vs urban. Table S4. Regression analysis of factors associated with outpatient service use using NB model: urban vs. rural. Table S5. Regression analysis of factors associated with inpatient service use using logit model: urban vs. rural. Table S6. Regression analysis of factors associated with OOP health expenditure using a two-part model combining logit regression and GLM: urban vs. rural. Table S7. Logit regression analysis of factors associated with the likelihood of incurring CHE: urban vs. rural.

\section{Abbreviations \\ AIC: Akaike Information Criterion; ALP: Affordability Ladder Program; BIC: Bayesian Information Criterion; CHE: Catastrophic Health Expenditure; Cl: Confidence Interval; GLM: Generalized Linear Model; NB: Negative Binomial; NCD: Non-communicable disease; NRCMS: New Rural Cooperative Medical Scheme; OOP: Out-of-pocket; SES: Socio-economic Status; UEBMI: Urban Employee Basic Medical Insurance; URBMI: Urban Resident Basic Medical Insurance; ZINB: Zero-inflated Negative Binomial; ZIP: Zero- inflated Poisson}

\section{Acknowledgements}

The authors of the paper express sincere gratitude to the Health Statistics and Information Center of Jiangsu Health Commission for the overall coordination during the implementation of the research project. The authors also gratefully acknowledge the Health Commission and the community health care workers in the primary health care centers in the two study sites for organizing the surveys and interviewing the participants, and the community members who gave their time to participate in the surveys.

\section{Authors' contributions}

The study was designed by LY, XX, WJ, LX and YZ. LY, XX, LX and YZ organized and coordinated the data collection process, and WJ participated in the data collection. XX and WJ cleared the dataset. WJ conducted

literature search and wrote the manuscript as the first author. ST, FC, CE and LY provided suggestions on data analysis framework and data interpretation, and helped revise the draft. All authors reviewed the draft manuscript and provided comments on the finalization of the manuscript. All authors have read and approved the manuscript in its current state.

\section{Funding}

The whole study was funded by the National Health Commission China. The roles of the funding agency mainly include providing support in the coordination of data collection in the study sites, and providing comments on the revision of the manuscript.

\section{Availability of data and materials}

The datasets generated and/or analyzed during the current study are not publicly available due to the fact that the data are owned by the National Health Commission China. The data could be available after obtaining consent from the National Health Commission China.

\section{Ethics approval and consent to participate}

This study was approved by the Institutional Review Board of Duke University. It was only conducted in China, and the local legislation does not require additional ethics approval in addition to the ethics approval from Duke University. Written informed consent was sought from and signed by all interviewed respondents.

\section{Consent for publication}

Anonymized information was used in this manuscript to ensure confidentiality.

\section{Competing interests}

The authors declare that they have no competing interests.

\section{Author details}

${ }^{1}$ Global Health Research Center, Duke Kunshan University, Kunshan 215316, China. ${ }^{2}$ School of Public Health, The University of Queensland, Brisbane 4006, Australia. ${ }^{3}$ Duke Global Health Institute, Duke University, Durham, NC 27710, USA. ${ }^{4}$ Health human Resources Development Center, National Health Commission, Beijing 100810, China. ${ }^{5}$ Center for Health Statistics and Information, National Health Commission, Beijing 100810, China. ${ }^{6}$ School of Business and Economics, Vrije Universiteit Amsterdam, 1081 HV Amsterdam, Netherlands. ${ }^{7}$ Department of Global Health and Amsterdam Institute for Global Health and Development, Amsterdam University Medical Centers, 1105 BP Amsterdam, Netherlands. ${ }^{8}$ Global Health Research Center, Duke Kunshan University, No. 8 Duke Avenue, Kunshan 215316, China.

Received: 31 July 2019 Accepted: 29 November 2019

Published online: 16 December 2019

References

1. Zhang X, Xiong Y, Ye J, Deng Z, Zhang X. Analysis of government investment in primary healthcare institutions to promote equity during the three-year health reform program in China. BMC Health Serv Res. 2013.

2. Li L, Fu H. China's health care system reform: Progress and prospects. Int J Health Plann Manag [Internet]. 2017;32(3):240-53 Available from: http://doi. wiley.com/10.1002/hpm.2424.

3. Chen G, Liu GG, Xu F. The impact of the urban resident basic medical insurance on health services utilisation in China. Pharmacoecon. 2014;32(3): 277-92.

4. Yang W. Catastrophic Outpatient Health Payments and Health Paymentinduced Poverty under China's New Rural Cooperative Medical Scheme. Appl Econ Perspect Policy [Internet]. 2015;37(1):64-85 Available from: https://academic.oup.com/aepp/article-lookup/doi/10.1093/aepp/ppu017.

5. Sun J, Deng S, Xiong X, Tang S. Equity in access to healthcare among the urban elderly in China: does health insurance matter? Int J Health Plann Manag. 2014;29(2):127-44. 
6. Liu GG, Zhao Z, Cai R, Yamada T, Yamada T. Equity in health care access to: assessing the urban health insurance reform in China. Soc Sci Med. 2002; 55(10):1779-94.

7. X. L, S. T, B. Y, N.K. P, F. Y, D.D. T, et al. Can rural health insurance improve equity in health care utilization? A comparison between China and Vietnam. Int J Equity Health [Internet]. 2012;11(1):1-9. Available from: http://ovidsp. ovid.com/ovidweb.cgi?T=JS\&PAGE=reference $\& D=$ =med10\&NEWS=N\&AN= 2012234220

8. Zhou Z, Su Y, Gao J, Campbell B, Zhu Z, Xu L, et al. Assessing equity of healthcare utilization in rural China: results from nationally representative surveys from 1993 to 2008. Int J Equity Health [Internet]. 2013;12(1):34 Available from: http://www.pubmedcentral.nih.gov/articlerender.fcgi?artid= 3673871\&tool=pmcentrez\&rendertype $=$ abstract $\% 5 \mathrm{Cn}$, http://www. equityhealthj.com/content/12/1/34

9. World Bank. The path to integrated insurance system in China (Vol. 2) : Main report (English). China health policy notes; no. 3. Washington, DC: World Bank. 2010. Available from: http://documents.worldbank.org/curated/en/926 821468024660940/Main-report.

10. Tang SL, Meng QY, Chen L, Bekedam H, Evans T, Whitehead M. Health system reform in China 1 tackling the challenges to health equity in China. Lancet. 2008:372(9648):1493-501.

11. Hu S, Tang S, Liu Y, Zhao Y, Escobar ML, de Ferranti D. Reform of how health care is paid for in China: challenges and opportunities. Lancet [Internet]. 2008;372(9652):1846-53 Available from: https://doi.org/10.1016/ S0140-6736(08)61368-9.

12. Chen M, Palmer AJ, Si L. Improving equity in health care financing in China during the progression towards universal health coverage. BMC Health Serv Res. 2017;17(1):1-8.

13. Pan J, Tian S, Zhou Q, Han W. Benefit distribution of social health insurance: evidence from china's urban resident basic medical insurance. Health Policy Plan. 2016;31(7):853-9.

14. Minchao J. Health Insurance in Urban China: disparity between peasant workers and legal urban residents. Int J Health Wellness [Internet]. 2011;1(1): 69-78 Available from: https://ezp.lib.unimelb.edu.au/login?url=https://search. ebscohost.com/login.aspx?direct=true\&db=a9h\&AN=66384715\&site=eds-live.

15. Yang W. China's new cooperative medical scheme and equity in access to health care: evidence from a longitudinal household survey. Int J Equity Health [Internet]. 2013;12:20. Available from: http://www.pubmedcentral.nih. gov/articlerender.fcgi?artid=3616826\&tool=pmcentrez\&rendertype=abstract

16. Zhang X, Dupre ME, Qiu L, Zhou W, Zhao Y, Gu D. Urban-rural differences in the association between access to healthcare and health outcomes among older adults in China. BMC Geriatr. 2017;17(1):1-11.

17. Fu R, Wang Y, Bao H, Wang Z, Li Y, Su S, et al. Trend of urban-rural disparities in hospital admissions and medical expenditure in China from 2003 to 2011. PLoS ONE. 2014:9(9).

18. Li J, Shi L, Liang H, Ding G, Xu L. Urban-rural disparities in health care utilization among Chinese adults from 1993 to 2011. BMC Health Serv Res. 2018;18(1):1-9.

19. Wang L, Wang A, Zhou D, FitzGerald G, Ye D, Jiang Q. An empirical analysis of rural-urban differences in out-of-pocket health expenditures in a lowincome society of China. PLoS One. 2016;11(5):1-12.

20. Cai J, Coyte PC, Zhao H. Decomposing the causes of socioeconomic-related health inequality among urban and rural populations in China: a new decomposition approach. Int J Equity Health. 2017;16(1):1-14.

21. Yeager RD, Yeager RD. Micropolitics in Rural Africa Linked references are available on JSTOR for this article. Micropolitics Rural Afr. 2019;3(1):81-6.

22. Dahlgren $\mathrm{G}$, Whitehead M. A framework for assessing health systems from the public's perspective: the ALPS approach. Int J Health Serv [internet]. 2007;37(2):363-78. Available from: http://www.ncbi.nlm.nih.gov/pubmed/1 7665729

23. Gilson L, Mclntyre D. Post-apartheid challenges: household access and use of health care in South Africa. Int J Health Serv [Internet]. 2007;37(4):673-91.

24. Iyer A, Sen G, George A. The dynamics of gender and class in access to health care: evidence from rural Karnataka, India. Int J Health Serv [Internet]. 2007:37(3):537-54

25. Luong DH, Tang S, Zhang T, Whitehead M. Vietnam during economic transition: a tracer study of health service access and affordability. Int $J$ Health Serv [internet]. 2007;37(3):573-88 Available from: http://www.ncbi. nlm.nih.gov/pubmed/17844935.

26. Jiangsu Government Report 2016 [Internet]. Available from: http://cn. chinagate.cn/reports/2016-03/05/content_37944056.htm
27. Culyer AJ, Wagstaff A. Equity and equality in health and health care. $J$ Health Econ. 1993;12(4):431-57.

28. Janicke DM, Finney JW, Riley AW. Children 's Health Care Use A Prospective Investigation of Factors Related to Care-Seeking. Med Care. 2018;39(9):9901001 Riley Published by : Lippincott Williams \& Wilkins Stable. https://www jstor.org/stable/3767778

29. Ranson MK. Reduction of catastrophic health care expenditures by a community-based health insurance scheme in Gujarat, India: Current experiences and challenges [Internet], Bulletin of the World Health Organization, vol. 80. GENEVA 27: WORLD HEALTH ORGANIZATION; 2002. p. 613-21

30. Neelon B, O'Malley AJ, Smith VA. Modeling zero-modified count and semicontinuous data in health services research part 1: background and overview. Stat Med. 2016;35(27):5070-93.

31. Neelon B, O'Malley AJ, Smith VA. Modeling zero-modified count and semicontinuous data in health services research part 2: case studies. Stat Med. 2016;35(27):5094-112.

32. Liang X, Guo H, Jin C, Peng X, Zhang X. The effect of new cooperative medical scheme on health outcomes and alleviating catastrophic health expenditure in China: A systematic review. PLoS ONE. 2012;7(8).

33. Liu P, Guo W, Liu H, Hua W, Xiong L. The integration of urban and rura medical insurance to reduce the rural medical burden in China: a case study of a county in Baoji City. BMC Health Serv Res. 2018;18(1):1-9.

34. Yang $X$, Chen $M, D u J$, Wang $Z$. The inequality of inpatient care net benefit under integration of urban-rural medical insurance systems in China. Int J Equity Health. 2018;17(1):1-12.

35. Shan L, Zhao M, Ning N, Hao Y, Li Y, Liang L, et al. Dissatisfaction with current integration reforms of health insurance schemes in China: are they a success and what matters? Health Policy Plan. 2018;33(3):345-54.

36. Xin W, Ang Z, Xin H, HangHang J. Integration of rural and urban healthcare insurance schemes in China: an empirical research. BMC Health Serv Res [Internet]. 2014;14(142):1-10 Available from: http://www.biomedcentral.com/ content/pdf/1472-6963-14-142.pdf.

37. Wang HQ, Liu ZH, Zhang YZ, Luo ZJ. Integration of current identity-based district-varied health insurance schemes in China: implications and challenges. Front Med Chin. 2012;6(1):79-84.

38. Meng Q, Fang H, Liu X, Yuan B, Xu J. Consolidating the social health insurance schemes in China: towards an equitable and efficient health system. Lancet. 2015:386(10002):1484-92.

39. Report series on the achievements in socio-ecnomic development 40 years since the reform opening-up policy in China [Internet]. 2018. Available from: http://www.stats.gov.cn/ztjc/ztfx/ggkf40n/201809/t20180903_1620407.html

\section{Publisher's Note}

Springer Nature remains neutral with regard to jurisdictional claims in published maps and institutional affiliations.
Ready to submit your research? Choose BMC and benefit from:

- fast, convenient online submission

- thorough peer review by experienced researchers in your field

- rapid publication on acceptance

- support for research data, including large and complex data types

- gold Open Access which fosters wider collaboration and increased citations

- maximum visibility for your research: over $100 \mathrm{M}$ website views per year

At BMC, research is always in progress.

Learn more biomedcentral.com/submissions 